\title{
Infecciones invasivas por Streptococcus pneumoniae en un hospital pediátrico de tercer nivel antes de la introducción de la vacuna conjugada. Características clínicas y serotipos involucrados
}

Invasive infections caused by Streptococcus pneumoniae in a terciary care pediatric hospital before the introduction of the conjugate vaccine. Clinical characteristics and serotypes involved

\author{
Dra. Guadalupe M. Péreza, Dra. Adriana Parra ${ }^{a}$, Dra. Lidia Casimir ${ }^{b}$, \\ Bca. Alejandra Mastroianni ${ }^{b}$, Bca. Vanesa Reijtman ${ }^{b}$, Dr. Horacio Lopardo ${ }^{b}$ y \\ Dra. Rosa Bologna ${ }^{a}$
}

\section{RESUMEN}

Las enfermedades invasivas por neumococo constituyenlaprincipalcausademorbimortalidad en los niños. En el Hospital "Prof. Dr. Juan P. Garrahan" se estudiaron retrospectivamente todas las infecciones invasivas por neumococo con hemocultivos positivos entre el 1 de octubre de 2008 y el 30 de septiembre de 2011, antes de la vacunación universal con la vacuna conjugada de 13 serotipos. Se identificaron 124 pacientes, con una media de edad de 48,3 meses (r: 1-216). El $58,9 \%$ de la población era mayor de 2 años (n: 73) y el $89 \%$ (n: 65) de ellos tenían una enfermedad de base. La principal forma de presentación fue la neumonía. Los serotipos de S. pneumoniae más frecuentes fueron: $14(22,5 \%, \mathrm{n}$ : 25$), 6(14,4 \%, \mathrm{n}$ : 16), 19 (8,1\%, n: 9), 23 (7,2\%, n: 8), 1 (6,3\%, n: 7), $5(4,5 \%, n: 5)$ y $7(7,2 \%, n: 8)$.

El 82,8\% de los serotipos de S. pneumoniae de esta serie están incluidos en la vacuna conjugada de 13 serotipos. Es esencial mantener la vigilancia epidemiológica para identificar la evolución y epidemiología de la enfermedad invasiva por neumococo en la Argentina.

Palabras clave: enfermedad neumocócica, Streptococcus pneumoniae, serotiposdeneumococo, neumonía, bacteriemia.

a. Servicio de Control Epidemiológico e Infectología.

b. Servicio de Microbiología. Hospital Nacional de Pediatría "Prof. Dr. Juan P. Garrahan".

Correspondencia: Dra. Guadalupe M. Pérez: guaperez@ hotmail.com

Conflicto de intereses: Ninguno que declarar.

Recibido: 24-8-2012 Aceptado: 2-1-2013 una estrategia eficaz para disminuir la incidencia de la enfermedad invasiva, tanto en los niños como en los adultos. ${ }^{5}$ En la Argentina se incorporó recientemente al calendario nacional de inmunizaciones la vacuna conjugada de 13 serotipos contra S. pneumoniae. ${ }^{6} \mathrm{El}$ presente estudio describe las características clínicas y los serotipos involucrados en las infecciones invasivas por neumococo documentados en hemocultivos en el Hospital de Pediatría "Prof. Dr. Juan P. Garrahan" en los tres años anteriores a la incorporación de la vacuna.

\section{MATERIAL Y MÉTODOS}

Se estudiaron retrospectivamente las infecciones invasivas por $S$. pneumoniae desde octubre de 2008 hasta septiembre de 2011 en el Hospital Garrahan, hospital pediátrico de alta complejidad, de derivación nacional, con 620 camas de internación y 4 unidades de cuidados intensivos. Se incluyeron todos los niños con un cuadro clínico compatible y con hemocultivos positivos para S. pneumoniae documentados en el hospital durante el período evaluado. Se excluyeron los pacientes en quienes el hallazgo de los hemocultivos se hizo en otra institución. Se revisaron las historias clínicas para registrar los antecedentes, y las características clínicas y evolutivas de cada paciente. Los resultados se enviaron a publicar 
garantizando la confidencialidad de la identidad de los pacientes. El estudio se llevó a cabo de acuerdo con los lineamientos éticos señalados en la Declaración de Helsinki. La serotipificación de los 111 aislamientos de neumococos disponibles en el momento del estudio se realizó en el INEIANLIS “Dr. Carlos G. Malbrán" por la reacción de Quellung, utilizando antisueros provistos por el Statens Serum Institut, Copenhague, Dinamarca.

\section{RESULTADOS}

Entre octubre de 2008 y septiembre de 2011 se identificaron 124 pacientes que tenían crecimiento de S. pneumoniae en los hemocultivos y un cuadro clínico compatible con enfermedad invasiva por neumococo. El 50,8\% de ellos (n: 63) eran varones. La media de edad fue de 48,3 meses (r: 1 a 216). El $67,7 \%$ (n: 84) tenían una enfermedad subyacente. Las comorbilidades que predominaron fueron: enfermedad hematooncológica 44,4\% (n: 36), síndrome nefrótico 9,9\% (n: 8), inmunodeficiencias $12,8 \%$ (n: 10) y cardiopatías congénitas 6,2\% (n: 5). Sólo 10 pacientes $(8,1 \%)$ tenían aplicada alguna vacuna contra el neumococo. La mortalidad fue del 9,7\% (n: 12).

Se analizaron las características de los pacientes según fueran de $\geq 2$ años o $<$ de 2 años (Tabla 1). Los $\geq 2$ años fueron el $58,9 \%$ de la población (n: 73), 89\% (n: 65) de ellos tenían una enfermedad de base. Los pacientes con enfermedad de base predominaron en el grupo de $\geq 2$ años $(p<0,01)$.

La principal forma de presentación de la enfermedad invasiva por S. pneumoniae fue la neumonía: 60,8\% (n: 31 ) de los menores de 2 años y $49,3 \%$ (n: 36) de los mayores de esa edad. En ambos grupos, 30\% presentaron sepsis. En el 5,9\% (n: 3 ) de los menores de 2 años y en el 13,7\% (n: 10) de los mayores el diagnóstico fue peritonitis primaria.

Los serotipos de $S$. pneumoniae más frecuentes fueron: 14 (22,5\%, n: 25), 6 (14,4\%, n: 16), 19 (8,1\%, n: 9$), 23(7,2 \%, n: 8), 1(6,3 \%, n: 7), 5(4,5 \%, n: 5)$ y 7 (7,2\%, n: 8) (Figura 1).

\section{DISCUSIÓN}

La enfermedad invasiva por S. pneumoniae continúa siendo la principal causa de enfermedad grave en los niños de todo el mundo. ${ }^{1}$ Es la causa más frecuente de neumonía bacteriana adquirida en la comunidad en las poblaciones no vacunadas $^{7}$ y el agente etiológico de sepsis de la comunidad y de meningitis. En nuestro país, los estudios poblacionales estimaron una incidencia anual de la enfermedad invasiva similar a la de otros países antes de la implementación de la vacunación universal. ${ }^{8}$

La incidencia informada en la bibliografía de enfermedad invasiva por S. pneumoniae es mayor en los menores de 2 años y en los pacientes inmunodeprimidos. ${ }^{1}$ Sin embargo, en la serie del Hospital Nacional de Pediatría "Prof. Dr. Juan P. Garrahan" predominaron los mayores de 2 años, hecho probablemente relacionado con las características de la población del hospital (enfermos crónicos, pacientes con enfermedades oncohematológicas, inmunodeficiencias y cardiopatías).

En esta serie predominó la neumonía con supuración pleural o sin ella como forma de

TABla 1. Características demográficas, clinicas y evolutivas de la población según la edad

\begin{tabular}{lcccc}
\hline Característica & Total & Menores de 2 años & Igual o mayor a 2 años & $p^{*}$ \\
\hline$\%(n)$ & $100(124)$ & $41,1(51)$ & $58,9(73)$ & NS \\
Enfermedad de base \% (n) & $67,7(84)$ & $37,3(19)$ & $89(65)$ & $<0,01$ \\
Varones \% (n) & $50,8(63)$ & $51(26)$ & $50,7(37)$ & $\mathrm{NS}$ \\
Media de edad en meses (rango) & $48,3(1-216)$ & $10,7(1-22)$ & $74,6(24-216)$ & - \\
Cuadro clínico \% (n) & & & & $\mathrm{NS}$ \\
$\quad$ Fiebre sin foco & $16,9(21)$ & $11,8(6)$ & $20,5(15)$ & $\mathrm{NS}$ \\
$\quad$ Meningitis & $0,8(1)$ & - & $1,4(1)$ & $\mathrm{NS}$ \\
$\quad$ Neumonía (con o sin derrame) & $54(67)$ & $60,8(31)$ & $49,3(36)$ & - \\
$\quad$ Foco osteoarticular & $1,6(2)$ & $3,9(2)$ & - & $\mathrm{NS}$ \\
$\quad$ Peritonitis & $10,4(13)$ & $5,9(3)$ & $30,1(22)$ & $\mathrm{NS}$ \\
$\quad$ Sepsis & $29,8(37)$ & $29,4(15)$ & $9,6(7)$ & $\mathrm{NS}$ \\
Fallece \% (n) & $9,6(12)$ & $9,8(5)$ & &
\end{tabular}

${ }^{*} \chi^{2}$ con y sin corrección de Yates y prueba exacta de Fisher. 
presentación clínica. Le siguieron, en orden de frecuencia, la sepsis y la fiebre sin foco. Esto coincide con la bibliografía, en la que se describe la neumonía, seguida de bacteriemia oculta y sepsis como síndromes prevalentes. ${ }^{9}$ Dada la asociación con el síndrome nefrótico y las características de la enfermedad subyacente de la población estudiada, la peritonitis primaria fue también una forma de presentación frecuente en esta serie. La mortalidad fue más alta que la descrita en la literatura especializada. ${ }^{10}$ Cabe considerar al respecto que el $67,7 \%$ de los niños tenían comorbilidades asociadas, lo que favorece la presentación de las formas más graves de infección.

La reciente incorporación, para los niños menores de 2 años, de la vacuna conjugada contra neumococo de 13 serotipos en la Argentina constituye una estrategia eficaz evaluada en otros países para la disminución de la enfermedad invasiva ${ }^{11}$ y la portación nasofaríngea de $S$. pneumoniae. ${ }^{12}$

La vacuna neumocócica 13-valente incluye los antígenos capsulares de los serotipos 1, 3, 4, 5, 6A, 6B, 7F, 9V, 14, 18C, 19A, 19F y $23 \mathrm{~F} .{ }^{5} \mathrm{El} 82,8 \%$ de los serotipos de la serie presentada están incluidos en la vacuna. Si se analizan solo los niños menores de 2 años, el 85,6\% de los serotipos identificados en el presente estudio están cubiertos con la vacuna conjugada de 13 serotipos.

En los países donde se aplica la vacunación universal con la vacuna heptavalente desde hace años, se ha notado la emergencia de serotipos no vacunales ${ }^{13,14}$ y el cambio en las características clínicas de la enfermedad invasiva por S. pneumoniae. ${ }^{15}$ En nuestro país la vacunación universal a los niños menores de 2 años con vacuna conjugada de 13 serotipos contra neumococo se comenzó a implementar en 2012. Es necesario mantener la atenta vigilancia epidemiológica y el estudio permanente de la evolución de la incidencia y formas clínicas de la enfermedad neumocócica invasiva y los serotipos predominantes.

\section{BIBLIOGRAFÍA}

1. Paganini H. Infectología Pediátrica. $1^{\text {ra }}$ ed. Buenos Aires: Editorial Científica Interamericana; 2007. Págs. 975-85.

2. Kaplan SL, Mason EO Jr, Wald ER, Schutze GE, et al. Decrease of invasive pneumococcal infections in children among 8 children's hospitals in the United States after the introduction of the 7-valent pneumococcal conjugate vaccine. Pediatrics 2004;113:443-9.

3. Ferro Bricks L, Berezin E. Impact of pneumococcal conjugate vaccine on the prevention of invasive pneumococcal diseases. J Pediatr (Rio J) 2006;82(3):S67-74.

4. Pírez MC, Algorta G, Cedrés A, Sobrero H, et al. Impacto de la vacunación neumocócica universal en las hospitalizaciones por neumonía y meningitis en niños de Montevideo, Uruguay. Pediatr Infect Dis J 2011;8:669-74.

5. Lexau C, Lynfield R, Danila R, Pilishvili T, et al. Changing epidemiology of invasive pneumococal disease among older adults in the era of pediatric pneumococcal conjugate vaccine. JAMA 2005;294:2043-51.

6. Ministerio de Salud de la Nación Argentina. Resolución $502 / 2011$.

7. Bradley J, Byington C, Shah S, Alverson B, et al. The management of community-acquired pneumonia in infants and children older than 3 months of age: Clinical Practice Guidelines by the Pediatric Infectious Diseases Society and the Infectious Diseases Society of America. Clin Infect Dis 2011;53:617-30.

8. Tregnaghi M, Ceballos A, Rüttimann R, Ussher J, et al.

FIGURA 1. Frecuencia de serotipos tipificados: total, en menores y en mayores de 2 años

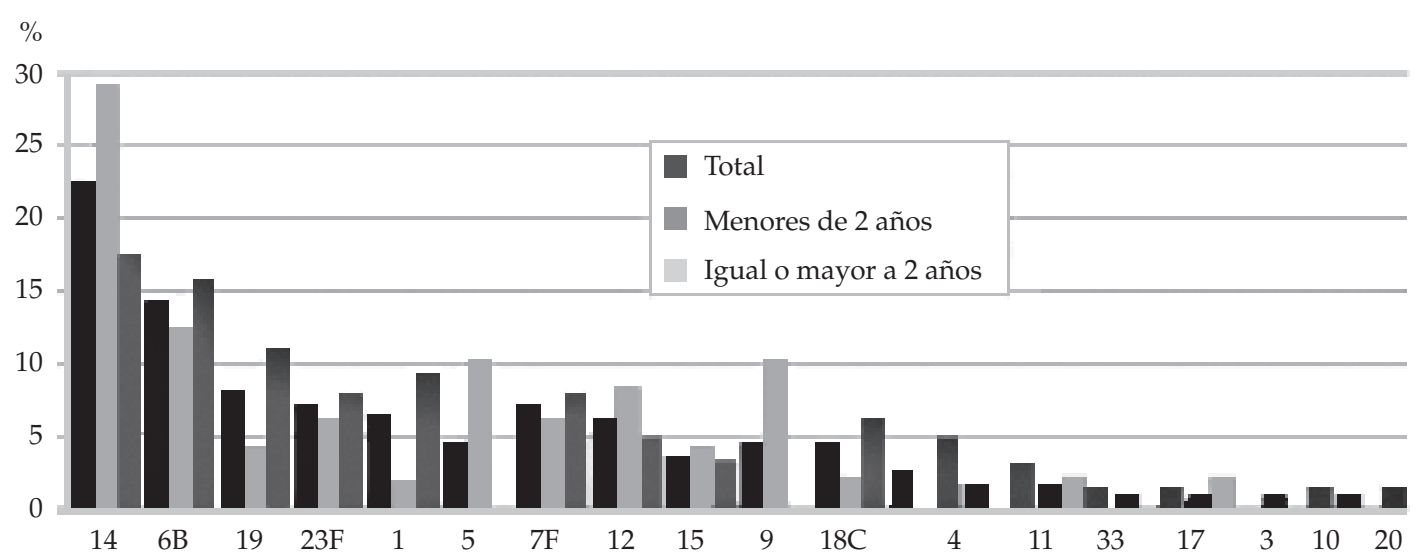


Active epidemiologic surveillance of pneumonia and invasive pneumococcal disease in ambulatory and hospitalized infants in Cordoba, Argentina. Pediatr Infect Dis J 2006;25:370-2.

9. Klugman KP, Feldman C. Streptococcus pneumoniae respiratory tract infections. Curr Opin Infect Dis 2001;14:173-9.

10. Christensen JS, Jensen TG, Kolmos HJ, Pedersen C, et al. Bacteremia with Streptococcus pneumoniae: sepsis and other risk factors for 30-day mortality-a hospitalbased cohort study. Eur J Clin Microbiol Infect Dis 2012;13(10):2719-25.

11. McIntosh EDG. How many episodes of hospital care might be prevented by widespread uptake of neumococcal conjugate vaccine? Arch Dis Child 2003;88:859-61.
12. Rinta-Kokko H, Dagan R, Givon-Lavi N, Auranen K. Estimation of vaccine efficacy against acquisition of pneumococcal carriage. Vaccine 2009;27:3831-7.

13. Kaplan S, Barson W, Lin P, Stovall S, et al. Infections in children serotype 19A is the most common serotype causing invasive pneumococcal. Pediatrics 2010;125:429-36.

14. de Sevilla MF, García-García JJ, Esteva C, Moraga F, et al. Clinical presentation of invasive pneumococcal disease in Spain in the era of heptavalent conjugate vaccine. Pediatr Infect Dis J 2012;31:124-8.

15. Ampofo K, Pavia AT, Chris S, Hersh AL, et al. The changing epidemiology of invasive pneumococcal disease at a tertiary children's hospital through the 7-valent pneumococcal conjugate vaccine era: a case for continuous surveillance. Pediatr Infect Dis J 2012;31:228-34.

\section{FE DE ERRATAS}

El artículo publicado en la sección Comunicación Breve en Arch Argent Pediatr 2013;111(2):128-139 titulado: Intervención familiar como complemento diagnóstico en el marco de la pediatría ampliada. Modelo del rompecabezas, la bibliografía debe figurar de la 1 a la 9 al final del artículo y no en el Anexo. 